\title{
Inflammatory myopathy in the context of an unusual overlapping laminopathy
}

1 UETeM - Molecular Pathology Group. IDIS-CIMUS, University of Santiago de Compostela, Spain 2 Division of Rheumatology, University Clinical Hospital of Santiago de Compostela, Spain ${ }^{3}$ Division of Pathology, University Clinical Hospital of Santiago de Compostela, Spain.

${ }^{4}$ Division of Endocrinology and Nutrition, University Clinical Hospital of Santiago de Compostela, Spain ${ }^{5}$ División of Neurology, University Clinical Hospital of Santiago de Compostela, Spain ${ }^{6}$ CIBER Fisiopatología de la Obesidad y la Nutrición (CIBERobn), Madrid, Spain

\section{Correspondence to:}

David Araújo-Vilar

UETeM, Department of Medicine,

IDIS CIMUS, Facultade de Medicina, University of Santiago de Compostela Avda de Barcelona s/n

15707 - Santiago de Compostela,

Spain

david.araujo@usc.es

Received on Jul/19/2017

Accepted on Feb/7/2018

DOI: $10.20945 / 2359-3997000000048$

\begin{abstract}
Cristina Guillín-Amarelle', Sofía Sánchez-lglesias' ${ }^{1}$, Antonio Mera², Elena Pintos ${ }^{3}$, Ana Castro-Pais ${ }^{4}$, Leticia Rodríguez-Cañete', Julio Pardo ${ }^{5}$, Felipe F. Casanueva ${ }^{4,6}$, David Araújo-Vilar ${ }^{1,4}$
\end{abstract}

\begin{abstract}
SUMMARY
Laminopathies are genetic disorders associated with alterations in nuclear envelope proteins, known as lamins. The $L M N A$ gene encodes lamins $\mathrm{A}$ and $\mathrm{C}$, and $L M N A$ mutations have been linked to diseases involving fat (type 2 familial partial lipodystrophy [FPLD2]), muscle (type 2 EmeryDreifuss muscular dystrophy [EDMD2], type 1B limb-girdle muscular dystrophy [LGMD1B], and dilated cardiomyopathy), nerves (type 2B1 Charcot-Marie-Tooth disease), and premature aging syndromes. Moreover, overlapping syndromes have been reported. This study aimed to determine the genetic basis of an overlapping syndrome in a patient with heart disease, myopathy, and features of lipodystrophy, combined with severe metabolic syndrome. We evaluated a 54-year-old woman with rheumatoid arthritis, chronic hypercortisolism (endogenous and exogenous), and a history of cured adrenal Cushing syndrome. The patient presented with a complex disorder, including metabolic syndrome associated with mild partial lipodystrophy (Köbberling-like); mild hypertrophic cardiomyopathy, with Wolff-Parkinson- White syndrome and atrial fibrillation; and limb-girdle inflammatory myopathy. Mutational analysis of the $L M N A$ gene showed a heterozygous c.1634G>A (p. R545H) variant in exon 10 of LMNA. This variant has previously been independently associated with FPLD2, EDMD2, LGMD1B, and heart disease. We describe a new, LMNA-associated, complex overlapping syndrome in which fat, muscle, and cardiac disturbances are related to a $\mathrm{p} . \mathrm{R} 545 \mathrm{H}$ variant. Arch Endocrinol Metab. 2018;62(3):376-82
\end{abstract}

\section{INTRODUCTION}

$\mathrm{M}$ utations in the LMNAgene (NM_170707.2) have been associated with a broad spectrum of diseases (1), including type 2 familial partial lipodystrophy (FPLD2), LMNA-related metabolic syndrome, type 2 Emery-Dreifuss muscular dystrophy (EDMD2), type 1B limb-girdle muscular dystrophy (LGMDIB), conduction-system diseases and dilated cardiomyopathy (DCMlA), and progeroid syndromes. FPLD2 begins in women during puberty, with a phenotype of fat loss in the limbs and buttocks, fat accumulation in the face and neck, well-defined musculature, phlebomegaly, insulin resistance, atherogenic dyslipidemia, and high cardiovascular risk (2).

The differential diagnosis includes Cushing's syndrome and truncal obesity. Previous studies have described a LMNA-associated metabolic syndrome with a Köbberling-like fat distribution $(3,4)$. EDMD2 is characterized as a progressive skeletal muscle weakness associated with early joint contractures.
LGMDIB causes muscular weakness in the spine and pelvic girdle (1).

Cardiac muscle laminopathies can present as nonspecific alterations in electrical conduction, or they can cause malignant arrhythmias and sudden death. Two of the most intriguing features of laminopathies are their clinical heterogeneity and the prevalence of overlapping syndromes. We investigated a female patient with a double Cushing syndrome (endogenous and exogenous), metabolic syndrome, cardiomyopathy, and limb-girdle muscular dystrophy. We aimed to determine whether this syndrome was related to a LMNA mutation.

\section{CASE AND METHODS}

This study was approved by the Ethics Review Panel of the Xunta de Galicia. The patient and her relatives provided informed consent for participation in the study and for publication of their clinical, biochemical, and genetic information. 


\section{Patient clinical history}

The patient (Table 1) was a 45-year-old female diagnosed with rheumatoid arthritis at age $40 \mathrm{y}$ and treated with corticosteroids from that point. She was referred to the Endocrinology Division due to high blood pressure, mixed dyslipidemia, and newly diagnosed diabetes (glycated hemoglobin: 9.6\%; systolic blood pressure: $150 \mathrm{mmHg}$; diastolic blood pressure: $90 \mathrm{mmHg}$; low-density lipoprotein cholesterol: $5.2 \mathrm{mmol} / \mathrm{L}$; high-density lipoprotein cholesterol: $0.65 \mathrm{mmol} / \mathrm{L}$; triglycerides: $3.42 \mathrm{mmol} / \mathrm{L})$. The patient had had an android appearance since puberty, with mild lipoatrophy in the limbs and buttocks and fat accumulation in the abdomen and face (Figure 1). The patient reported that her deceased father had striking hypermuscular limbs, prominent abdominal fat, and diabetes. He had undergone a pacemaker implantation because of a third-degree atrioventricular block.

At age $46 \mathrm{y}$, the patient displayed poor glycemic control, and glucocorticoids were discontinued for several months, with no improvement. She was then diagnosed with adrenal Cushing's syndrome, based on a 34- $\mathrm{mm}$ right adrenal adenoma (urinary free cortisol: $1100 \mu \mathrm{g} / 24 \mathrm{~h}$ ), which was histologically confirmed after a laparoscopic adrenalectomy. Additional hydrocortisone replacement was necessary, and the patient currently continues this treatment. After the adrenal tumor was resected, glycemic control, blood pressure, and lipid levels improved. She continued pharmacological treatment with antihypertensive drugs and metformin, but she was able to discontinue insulin.

Some months after the adrenalectomy, the patient was diagnosed with Wolff-Parkinson-White syndrome associated with atrial fibrillation, due to preexcitation, which was successfully ablated with radiofrequency. Transthoracic ultrasonography revealed a slightly hypertrophic non-dilated left ventricle, with preserved systolic function, type 1 diastolic dysfunction, and mild mitral regurgitation. No coronary lesions were detected with cardiac catheterization.

At age $50 \mathrm{y}$, the patient complained of muscular weakness without pain or contractures.

Table 1. Chronological evolution of clinical data

\begin{tabular}{|c|c|c|c|c|c|}
\hline Age (years) & 45 & 46 & 47 & 48 & $49-52$ \\
\hline \multirow[t]{7}{*}{ Event } & & Adrenal & & & \\
\hline & Diabetes. Start & Cushing. & & Adrenal & \\
\hline & antidiabetic & Start statins & & surgery, & Myopathy. \\
\hline & and & and insulin; & & start & Stop statins \\
\hline & antihypertensi & intensive & & hydrocortiso & at 49 \\
\hline & ve drugs & antihypertensi & & ne & \\
\hline & & ve therapy & & & \\
\hline BMI & 30.5 & 31.7 & 30.8 & 36.5 & $25-26$ \\
\hline \multirow[t]{2}{*}{$\mathrm{BP}(\mathrm{mmHg})$} & $150 / 90$ & $165 / 109$ & $130 / 9$ & $140 / 93$ & $125 / 80$ \\
\hline & & & 0 & & \\
\hline CPK (UI/L) & - & 176 & 88 & 157 & $378-2500$ \\
\hline LDH (U/L) & - & - & - & & $556-860$ \\
\hline Glucose (mg/dL) & 250 & 331 & 236 & 137 & $65-91$ \\
\hline $\mathrm{HbA1C}(\%)$ & - & 9.6 & 10.2 & 8.1 & $6-6.1$ \\
\hline Triglycerides (mg/ dL) & 164 & 122 & 146 & 254 & $202-214$ \\
\hline $\mathrm{HDL}(\mathrm{mg} / \mathrm{dL})$ & - & 37 & 41 & 30 & $40-40$ \\
\hline LDL (mg/dL) & - & 161 & 126 & - & $63-139$ \\
\hline Leptin ( $\mu g / L)$ & - & - & 12.7 & 9.9 & $1-8.4$ \\
\hline Insulin* (mUI/L) & - & 46.5 & - & - & 43.9 \\
\hline UFC (Ug/24h) & - & 838 & 1014 & - & 36 \\
\hline Cortisol** (ug/dL) & 23 & 30 & 27.4 & 2.2 & 1.6 \\
\hline ACTH & - & 13 & 9 & - & 60 \\
\hline
\end{tabular}

BP: blood pressure; UFC: urinary free cortisol; *: without exogenous insulin: **: stopping oral corticoid almost $24 \mathrm{~h}$ before. 


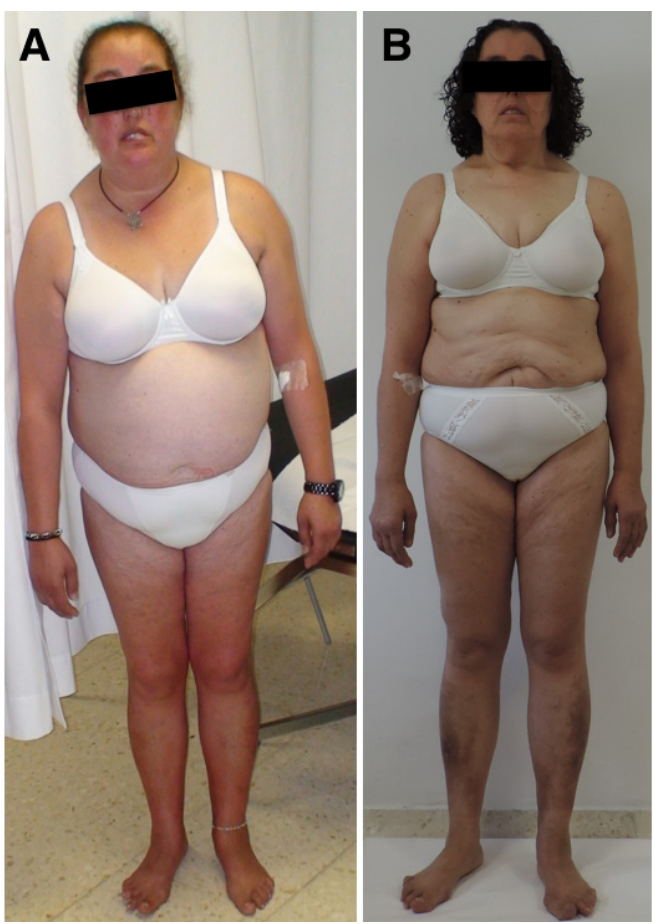

Figure 1. Photographs of the patient show body morphology due to a LMNA variant. (A) Before receiving a cure for Cushing's syndrome (46 years old). (B) Six years after receiving a cure for Cushing's syndrome (54 years old).
Creatine kinase levels ranged from 321 to 2525 $\mathrm{IU} / \mathrm{L}$, and high levels persisted after discontinuation of statins. On physical examination, muscle weakness was evident, predominantly in the pelvic girdle. She was unable to get up from the ground and had marked difficulty in rising from a chair without hand support. Electromyoneurography revealed myopathy changes and spontaneous activity (fibrillation and positive waves) in proximal muscles, without polyneuropathy.

\section{Body composition}

Skinfolds (Table 2) were measured in triplicate on the dominant extremity with a Lange skinfold caliper (Cambridge Scientific Industries, MD, USA). Segmental body fat distribution was assessed with whole-body dual-energy X-ray absorptiometry, performed with a Lunar model DPX apparatus (GE Medical Systems, Milwaukee, WI, USA).

\section{Molecular analyses}

DNA was prepared from peripheral white blood cells following standard procedures (5). LMNA exons $1-12$

Table 2. Changes in body composition before and after Cushing cure evaluated by anthropometry and DXA

\begin{tabular}{|c|c|c|c|c|}
\hline & Active cushing & Cured cushing & Obese control* & Non obese control* \\
\hline Age & 46 & 48.6 & & \\
\hline Weight (kg) & 82.2 & 65.3 & & \\
\hline Height (cm) & 161 & 161 & & \\
\hline BMI $\left(\mathrm{kg} / \mathrm{m}^{2}\right)$ & 31.7 & 25.21 & $38.1 \pm 6.5$ & $26.6 \pm 2.8$ \\
\hline Tricipital skinfold (mm) & 20 & 17 & $38.8 \pm 10.0$ & $29.4 \pm 11.7$ \\
\hline Bicipital skinfold (mm) & 15 & 10 & $32.4 \pm 11.6$ & $21.5 \pm 11.8$ \\
\hline Suprailiac skinfold (mm) & 42 & 15 & $52.2 \pm 14.7$ & $30.6 \pm 14.4$ \\
\hline Subescapular skinfold (mm) & 35 & 18 & $44.2 \pm 12.3$ & $28.4 \pm 16.4$ \\
\hline Thigh skinfold (mm) & 19 & 10 & $41.1 \pm 14.5$ & $22.7 \pm 8.6$ \\
\hline Calf skinfold (mm) & 6 & 5 & $18.9 \pm 14.2$ & $11.2 \pm 4.8$ \\
\hline WHR & 1.0 & 0.93 & $0.89 \pm 0.08$ & $0.88 \pm 0.06$ \\
\hline Total fat (kg) & 37.5 & 20.06 & $43.8 \pm 11.7$ & $26.4 \pm 7.7$ \\
\hline Total fat (\%) & 46.1 & 30.6 & $48.2 \pm 6.4$ & $39.3 \pm 7.0$ \\
\hline Upper limbs fat (kg) & 3.48 & 2.26 & $4.44 \pm 1.1$ & $3.1 \pm 1.0$ \\
\hline Upper limbs fat (\%) & 45.3 & 31.6 & $47.7 \pm 5.7$ & $42.1 \pm 7.3$ \\
\hline Lower limbs fat (kg) & 9.69 & 6.16 & $12.4 \pm 3.8$ & $8.4 \pm 2.2$ \\
\hline Lower limbs fat (\%) & 40.8 & 30.5 & $44.6 \pm 6.4$ & $39.8 \pm 6.4$ \\
\hline Trunk fat (kg) & 23.2 & 10.84 & $22.9 \pm 5.1$ & $14.0 \pm 5.7$ \\
\hline Trunk fat (\%) & 51.7 & 31.8 & $52.5 \pm 7.6$ & $40.8 \pm 10.2$ \\
\hline Visceral fat (g) & ND & 764 & $2012 \pm 894$ & $992 \pm 693$ \\
\hline Trunkal/Lower limbs fat ratio (kg) & 2.39 & 1.75 & 1.61 & 1.66 \\
\hline
\end{tabular}

*: Normal ranges from ref. 3; WHR: waist to hip ratio. 
and the surrounding intronic sequences were amplified by PCR. Primers and conditions have been previously described (6).

\section{Histological muscle studies}

Deltoid muscle biopsy samples were snap frozen in liquid nitrogen, and cryostat sections were processed and stained to exclude dystrophies and other pathological conditions (e.g., mitochondrial myopathies, metabolic diseases) as follows: PAS, modified Gomori trichrome, Oil Red O, enzyme histochemistry (muscle phosphorylase, muscle phosphofructokinase, lactate dehydrogenase, AMP deaminase, cytochrome-C oxidase, $\mathrm{SDH}$, and $\mathrm{NADH}$ ), and immunohistochemistry (beta-spectrin to check preservation of the plasma membrane; slow, fast, and fetal myosin; utrophin; alpha, beta, gamma, and delta sarcoglycan; dystrophin fractions: N- terminal, C-terminal, and rod domain; NOS; alpha and beta dystroglycan; dysferlin, caveolin-3, collagen type VI, laminin alpha2 chain of merosin (laminin-2), telethonin, myotilin, desmin, calpain-3, MHC class I antigen, and inflammatory markers (CD68/KP1, CD3 and CD20 for histiocytes and lymphocytes). The only commercial antibodies available for Lamin A/C are not helpful for diagnostic purposes (here it was used as a positive control for emerin antibody).

Immunostains were performed after antigen retrieval using a standard avidin-biotin immunoperoxidase detection technique (EnVision Systems, Dako, Glostrup, Denmark).

\section{RESULTS}

We identified a heterozygous c.1634G $>$ A (p.R545H) variant of $L M N A$, located in exon 10. This variant had not been probed for pathogenicity with in silico approaches (PolyPhen and SIFT).

Its allelic frequency was 0.00019 (1000 Genomes Project). Of the patient's relatives whom we studied (mother, sisters, offspring), none carried this variant. Histological findings of muscle samples were consistent with acute and chronic, nonspecific, inflammatory myopathy (Figure 2).

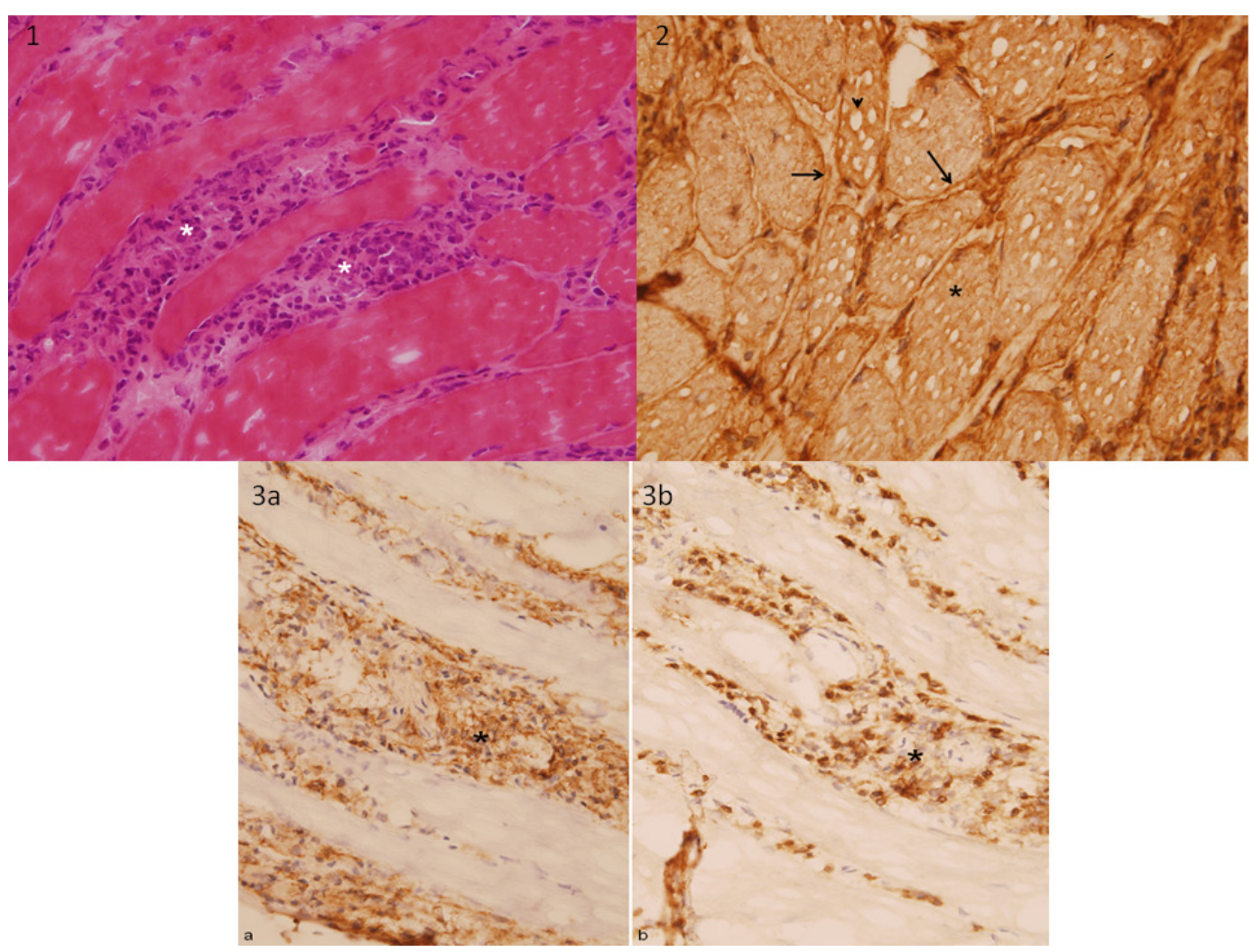

Figure 2. (1) Snap frozen cryostat sections of deltoid muscle showing endomisial inflammatory infiltrates composed mainly of hystiocytes (asterisks) (Hematoxylin and eosin, 400x). (2) MHC class I antigen is upregulated in all fibers, with immunolabeling at plasma membrane (arrow) and sarcoplasm (asterisk). See also "pseudovacuoles" secondary to "ice crystals" snap frozen artifact (arrow head) (Immunoperoxidase reaction, diaminobenzidine brown staining chromogen, 400x). (3a) Immunostaining of CD68 (KP1) antibody remark histiocityc inflammatory component between myofibers, with (3b) a minor population of CD3 antibody positive T lymphocytes (asterisks) (400x). 


\section{DISCUSSION}

We investigated an unusual case of laminopathy and found that it was due to a p.R545H LMNA variant that overlapped with chronic hypercortisolism. Thus, after chronic hypercortisolism had been diagnosed and cured, the patient exhibited atypical partial lipodystrophy, idiopathic inflammatory myopathy, and cardiomyopathy with conduction disturbances. Although this variant has not been shown to be pathogenic with in silico approaches, it has been associated with FPLD2 (7), EDMD2 (8), LGMD1B (9), and heart disease (10).

In FPLD2, more than $80 \%$ of cases are due to missense mutations in exon 8; however, atypical phenotypes have been related to mutations in other exons (11). Moreover, several patients with mutations outside the immunoglobulin-like fold of lamin A have lacked the typical FPLD2 phenotype but experienced insulin resistance (4). In addition, some cases of FPLD2 have been associated with heart conduction disorders, valvulopathies, and cardiomyopathy (12-14). Other authors have reported LMNA-associated complex phenotypes, including heart failure and limb-girdle muscular dystrophy, due to a Ser334del variant $(15,16)$; muscular dystrophy, lipodystrophy, and cardiac rhythm disturbances related to a R527P variant (17); or FPLD, early heart failure, first-degree atrioventricular block, and late proximal muscle weakness due to a $\mathrm{R} 28 \mathrm{~W}$ variant (12).

Exon 10 of $L M N A$ corresponds to the C-terminal domain, common to both lamins $\mathrm{A}$ and $\mathrm{C}$, which forms an immunoglobulin-like, three-dimensional structure (1). The conformation of this domain is well defined for residues $430-545$ (18). Arginine 545 is at the external surface of the structure. Mutations in the Ig-fold can affect either head-to-tail polymerization, which destabilizes the three-dimensional structure of the $\mathrm{C}$-terminal domain, or lamin $\mathrm{A} / \mathrm{C}$ interactions with other proteins (1). At least 13 missense mutations at the C-terminal domain are related to EDMD2 (18), including the nearby $\mathrm{R} 541 \mathrm{H}$. Although the R545 residue has not been specifically studied, it does not seem to influence the stability of the $3 \mathrm{D}$ structure. However, the exchange of arginine (positive polar) for histidine (neutral polar) could alter interactions with other proteins at the nuclear lamina.

This study was particularly challenging because of the presence of Cushing's syndrome and the autoimmune background. Chronic hypercortisolism causes a characteristic fat distribution, in particular, excess abdominal fat (19); however, no particular changes in limb fat have been reported (20). Strikingly, in this patient, once hypercortisolism was cured, an abnormal fat distribution became more evident, although it was not as severe as observed in classical Dunnigan disease. This change in fat distribution was particularly intriguing because it highlighted important differences among LMNA mutations that cause FPLD (13). The $\mathrm{R} 545 \mathrm{H}$ variant, previously associated with FPLD (7), caused severe metabolic syndrome with android fat distribution in this patient.

The patient was initially diagnosed with polymyositis, based on girdle weakness, high creatine kinase, and muscle lymphocyte infiltration. Because of her autoimmune background, this diagnosis was probably the most parsimonious. However, the $\mathrm{R} 545 \mathrm{H}$ variant has been associated with LGMDIB (9), which clinically overlaps with polymyositis. Other LMNA mutations that cause muscular and/or cardiac laminopathies have been related to inflammatory changes in muscle specimen biopsies. For example, in biopsies from patients with infantile- onset LMNAassociated myopathy, Komaki and cols. (21) reported mononuclear cell infiltrations that were positive for lymphocyte markers CD4, CD8, or CD20, active necrosis, and regeneration. Additionally, they observed elevated sarcolemmal HLA staining in many fibers. Similarly, HLA class I antigens were upregulated in our samples and in samples described previously in a study on LGMDIB $(15,16)$. It cannot be ruled out that chronic hypercortisolism had influenced the myopathy. However, there are some clues to differences between the corticoid-myopathy case and the case described here.

In the first case, serum values of muscle enzymes are typically normal or slightly elevated, electromyoneurography is usually normal, and there are no inflammatory infiltrates or necrosis on muscle biopsy samples (22). Although unusual, polymyositis has been associated with cardiac involvement, manifested as rhythm disturbances, conduction defects, and heart failure (23). Similarly, laminopathies, like FPLD2 or LGMDIB $(9,14)$, and particularly the $\mathrm{R} 545 \mathrm{H}$ variant, have also been related to heart disease (10). Patients with cardiac compromise related to LMNA typically show initial signs of nonspecific rhythm disturbances after age $30 \mathrm{y}(24)$, and they frequently need a 
permanent pacemaker. Later, cardiac compromise may be further complicated with cardiomyopathy (25). Our patient was diagnosed with Wolff-Parkinson-White syndrome, atrial fibrillation, mitral regurgitation, and mild hypertrophic cardiomyopathy. Taken together, the diagnoses of severe metabolic syndrome, girdle weakness appearing in the fourth decade, and heart involvement in a patient that carried the $\mathrm{R} 545 \mathrm{H}$ variant in $L M N A$ resembled a complex laminopathic disorder. However, the presence of chronic hypercortisolism represented a confounding factor, and rheumatoid arthritis prevented us from definitely ruling out a random association of different unrelated autoimmune entities.

In summary, this case emphasizes the need for actively searching for $L M N A$ mutations in patients with clinical features compatible with partial lipodystrophy, cardiac conduction abnormalities, cardiomyopathy, and/or certain types of myopathy. A correct diagnosis can facilitate early interventions to prevent the consequences of these pathologies, which can be lethal.

Acknowledgments: we are indebted to the patients of this study for their collaboration. This work was funded by the Instituto de Salud Carlos III (grant number: PI081449) and the European Regional Development Fund, FEDER. In addition, SRG was awarded a Research Fellowship granted by the Asociación Española de Familiares y Afectados de Lipodistrofias (AELIP). None of the authors has any conflict of interest to disclose.

Ethical Publication Statement: we confirm that we have read the Journal's position on issues involved in ethical publication and affirm that this report is consistent with those guidelines.

Disclosure: no potential conflict of interest relevant to this article was reported.

\section{REFERENCES}

1. Broers JL, Ramaekers FC, Bonne G, Yaou RB, Hutchison CJ. Nuclear lamins: laminopathies and their role in premature ageing. Physiol Rev. 2006;86(3):967-1008.

2. Brown RJ, Araujo-Vilar D, Cheung PT, Dunger D, Garg A, Jack $M$, et al. The Diagnosis and Management of Lipodystrophy Syndromes: A Multi-Society Practice Guideline. J Clin Endocrinol Metab. 2016;101(12):4500-11.

3. Guillin-Amarelle C, Sanchez-Iglesias S, Castro-Pais A, RodriguezCanete L, Ordonez-Mayan L, Pazos M, et al. Type 1 familial partial lipodystrophy: understanding the Kobberling syndrome. Endocrine. 2016;54(2):411-21.

4. Decaudain A, Vantyghem MC, Guerci B, Hecart AC, Auclair M, Reznik $Y$, et al. New metabolic phenotypes in laminopathies: LMNA mutations in patients with severe metabolic syndrome. J Clin Endocrinol Metab. 2007;92(12):4835-44.

5. Sambrook J, Russell DW. The Condensed Protocols from Molecular Cloning: A Laboratory Manual: Cold Spring Harbor Laboratory Press; 2006.
6. Araujo-Vilar D, Loidi L, Dominguez F, Cabezas-Cerrato J. Phenotypic gender differences in subjects with familial partial lipodystrophy (Dunnigan variety) due to a nuclear lamin A/C R482W mutation. Horm Metab Res. 2003;35(1):29-35.

7. Chan D, Mclntyre AD, Hegele RA, Don-Wauchope AC. Familial partial lipodystrophy presenting as metabolic syndrome. J Clin Lipidol. 2016;10(6):1488-91.

8. Huong S. Molecular genetic studies in hereditary laminopathies of man.: Ernst- Moritz-Arndt-Universität, Greifswald; 2010.

9. Maggi L, Carboni N, Bernasconi P. Skeletal Muscle Laminopathies: A Review of Clinical and Molecular Features. Cells. 2016;5(3):pii: E33.

10. van Rijsingen IA, Nannenberg EA, Arbustini E, Elliott PM, Mogensen J, Hermans-van Ast JF, et al. Gender-specific differences in major cardiac events and mortality in lamin $\mathrm{A} / \mathrm{C}$ mutation carriers. Eur J Heart Fail. 2013;15(4):376-84.

11. Garg A, Vinaitheerthan M, Weatherall PT, Bowcock AM. Phenotypic heterogeneity in patients with familial partial lipodystrophy (dunnigan variety) related to the site of missense mutations in lamin a/c gene. J Clin Endocrinol Metab. 2001;86(1): 59-65.

12. Garg A, Speckman RA, Bowcock AM. Multisystem dystrophy syndrome due to novel missense mutations in the amino-terminal head and alpha-helical rod domains of the lamin A/C gene. Am J Med. 2002;112(7):549-55.

13. Araujo-Vilar D, Lado-Abeal J, Palos-Paz F, Lattanzi G, Bandin MA, Bellido D, et al. A novel phenotypic expression associated with a new mutation in LMNA gene, characterized by partial lipodystrophy, insulin resistance, aortic stenosis and hypertrophic cardiomyopathy. Clin Endocrinol (Oxf). 2008;69(1):61-8.

14. Panikkath $R$, Panikkath D, Sanchez-Iglesias S, Araujo-Vilar D, Lado-Abeal J. An Uncommon Association of Familial Partial Lipodystrophy, Dilated Cardiomyopathy, and Conduction System Disease. J Investig Med High Impact Case Rep. 2016;4(3):2324709616658495.

15. Madej-Pilarczyk A, Niezgoda A, Janus M, Wojnicz R, Marchel M, Fidzianska $A$, et al. Limb-girdle muscular dystrophy with severe heart failure overlapping with lipodystrophy in a patient with LMNA mutation p.Ser334del. J Appl Genet 2017;58(1):87-91.

16. Madej-Pilarczyk A, Niezgoda A, Janus M, Wojnicz R, Marchel M, Fidziańska $A$, et al. Limb-girdle muscular dystrophy with severe heart failure overlapping with lipodystrophy in a patient with LMNA mutation p.Ser334del. J Appl Genet. 2017;58(1):87-91.

17. van der Kooi AJ, Bonne G, Eymard B, Duboc D, Talim B, Van der Valk $M$, et al. Lamin A/C mutations with lipodystrophy, cardiac abnormalities, and muscular dystrophy. Neurology. 2002;59(4):620-3.

18. Krimm I, Ostlund C, Gilquin B, Couprie J, Hossenlopp P, Mornon $\mathrm{JP}$, et al. The Ig-like structure of the C-terminal domain of lamin $\mathrm{A} / \mathrm{C}$, mutated in muscular dystrophies, cardiomyopathy, and partial lipodystrophy. Structure. 2002;10(6):811-23.

19. Garrapa GG, Pantanetti P, Arnaldi G, Mantero F, Faloia E. Body composition and metabolic features in women with adrenal incidentaloma or Cushing's syndrome. J Clin Endocrinol Metab. 2001;86(11):5301-6.

20. Rockall AG, Sohaib SA, Evans D, Kaltsas G, Isidori AM, Monson $\mathrm{JP}$, et al. Computed tomography assessment of fat distribution in male and female patients with Cushing's syndrome. Eur J Endocrinol. 2003;149(6):561-7.

21. Komaki H, Hayashi $Y K$, Tsuburaya R, Sugie K, Kato M, Nagai T, et al. Inflammatory changes in infantile-onset LMNA-associated myopathy. Neuromuscul Disord. 2011;21(8):563-8.

22. Gupta A, Gupta Y. Glucocorticoid-induced myopathy: Pathophysiology, diagnosis, and treatment. Indian J Endocrinol Metab. 2013;17(5):913-6.

23. Eisen A, Arnson Y, Dovrish Z, Hadary R, Amital H. Arrhythmias and conduction defects in rheumatological diseases--a comprehensive review. Semin Arthritis Rheum. 2009;39(3):145-56. 
24. van Berlo JH, de Voogt WG, van der Kooi AJ, van Tintelen JP, Bonne G, Yaou RB, et al. Meta-analysis of clinical characteristics of 299 carriers of LMNA gene mutations: do lamin A/C mutations portend a high risk of sudden death? J Mol Med (Berl). 2005;83(1):79-83.
25. Carboni N, Mateddu A, Marrosu G, Cocco E, Marrosu MG. Genetic and clinical characteristics of skeletal and cardiac muscle in patients with lamin $A / C$ gene mutations. Muscle Nerve. 2013;48(2):161-70. 